\title{
Short-Vessel Occlusion Might Indicate Higher Possibility of Success in Reperfusion following Mechanical Thrombectomy in Acute Middle Cerebral Artery Occlusion
}

\author{
Tomoyuki Yoshihara $^{a}$ Ryuzaburo Kanazawa $^{b}$ Takanori Uchida $^{b}$ \\ Tetsuhiro Higashida $^{\text {b Hidenori Ohbuchic Naoyuki Arai }{ }^{c} \text { Yuichi Takahashic }}{ }^{c}$ \\ ${ }^{a}$ Department of Emergency and Critical Care Medicine, Kansai Medical University General Medical Center, Osaka, \\ Japan; ${ }^{b}$ Department of Neurosurgery, Nagareyama Central Hospital, Chiba, Japan; ' Department of Neurosurgery, \\ Medical Center East, Tokyo Women's Medical University, Tokyo, Japan
}

\section{Keywords}

Flat-panel detector computed tomography · Maximum intensity projection - Middle cerebral artery $\cdot$ Reperfusion . Thrombectomy

\begin{abstract}
Background: The impact of the length of the occluded vessel in acute large-vessel occlusion on successful reperfusion by mechanical thrombectomy remains unclear. This study evaluated whether diameter and length of the occluded vessel in acute middle cerebral artery (MCA) occlusion might relate to successful reperfusion following mechanical thrombectomy. Methods: This retrospective study included patients with acute MCA occlusion who underwent intra-aortic injection of contrast medium to obtain maximum intensity projection (MIP) images acquired by flat-panel detector computed tomography (FD-CT) equipped with an angiographic system. All patients received mechanical thrombectomy and were divided into two groups: those with successful reperfusion (Thrombolysis in Cerebral Infarction [TICI] $2 \mathrm{~b} / 3$ ) and those without. We compared the diameter and length of the occluded vessel between the groups. In the sub-analysis of patients with stent retriever use, ratio of
\end{abstract}

karger@karger.com www.karger.com/cee

Karger"

BOPEN ACCESS
(C) 2021 The Author(s)

Published by S. Karger AG, Basel

This is an Open Access article licensed under the Creative Commons Attribution-NonCommercial-4.0 International License (CC BY-NC) (http://www.karger.com/Services/OpenAccessLicense), applicable to the online version of the article only. Usage and distribution for commercial purposes requires written permission. length of occluded vessel to length of the active zone was compared. Results: We enrolled 29 patients (median age: 73, M1 occlusion: $51 \%$, stent retriever use: $72 \%)$. Eighteen patients achieved $\mathrm{TICl} 2 \mathrm{~b} / 3$ with significantly larger distal end diameter (1.7 [interquartile range: 1.5-1.9] vs. 1.2 [1.2-1.5] $\mathrm{mm}, p=0.007)$ and shorter length (7.1 [4.9-9.7] vs. $12.3[7.2-$ $15.8] \mathrm{mm}, p=0.043$ ) of the occluded vessel. Sub-analysis of 21 patients showed that the cut-off value for $\mathrm{TICl} 2 \mathrm{~b} / 3$ reperfusion was 0.32 as the ratio between the occluded vessel and stent retriever active zone (receiver operating characteristic area under the curve: 0.90 ). Conclusion: In acute MCA occlusion, larger diameter of the distal end and shorter length of the occluded vessel on FD-CT MIP images might indicate a higher possibility of achieving $\mathrm{TICl} 2 \mathrm{~b} / 3$ following mechanical thrombectomy.

(c) 2021 The Author(s).

Published by S. Karger AG, Basel

\section{Introduction}

Rapid and safe reperfusion by mechanical thrombectomy contributes to good functional outcome following acute large-vessel occlusion in stroke [1]. Several predictive factors reported for successful reperfusion include 
thrombus location [2], vessel anatomy [3], and collateral flow [4]. However, how the length of the occluded vessel impacts success in reperfusion following mechanical thrombectomy remains unclear, although previous studies $[5,6]$ tried to evaluate the length of the occlusive thrombus for the primary purpose of differentially diagnosing cardiogenic embolism.

Recently, flat-panel detector computed tomography (FD-CT) equipped with an angiographic system has made it possible to evaluate perfusion studies without any loss of time in patients with acute ischemic stroke. The syngo DynaPBV Neuro (Siemens, Erlangen, Germany) provides cerebral PBV (parenchymal blood volume) mapping by FD-CT that correlates well with cerebral blood volume images derived from conventional CT perfusion studies [7]. Moreover, a volume-rendered image, which is reconstructed from a maximum intensity projection (MIP) image acquired by FD-CT, makes it possible to identify the proximal end of an occluded vessel in a manner equivalent to that by MR angiography [8].

In the present study, we measured the diameter and length of the occluded vessel on MIP images derived from raw data of the syngo DynaPBV Neuro in patients with acute occlusion of the middle cerebral artery (MCA). Then, we retrospectively studied whether these measured diameters and lengths of the occluded vessel might have relevancy to success in reperfusion following mechanical thrombectomy.

\section{Materials and Methods}

We included patients with acute occlusion of the MCA within $12 \mathrm{~h}$ of onset who had undergone examination by conventional angiography and syngo DynaPBV Neuro with the use of FD-CT from January 2016 to December 2017. On patient arrival, neurological deficits were examined according to the National Institutes of Health Stroke Scale. After exclusion of intracranial hemorrhage by head CT, brain MRI (diffusion-weighted imaging, fluid-attenuated inversion recovery, and magnetic resonance angiography) was performed to diagnose acute occlusion of the MCA (shown in Fig. 1a) and calculate the Alberta Stroke Program Early CT Score (ASPECTS)-diffusion-weighted imaging by the 11-point method. If applicable, $0.6 \mathrm{mg} / \mathrm{kg}$ alteplase was intravenously injected into the patients according to the "Guidelines for the intravenous application of recombinant tissue-type plasminogen activator (alteplase), the 2nd edition" advocated by the Japan Stroke Society [9]. MCA occlusion was identified by conventional internal carotid arteriography (shown in Fig. 1b). The MIP image was acquired without any loss of time by two rotations of FD-CT in reference to the DynaPBV Neuro mode as follows: the first run obtained the initial mask, and the second run obtained the contrast enhancement images. After the first run, $10 \%$ diluted contrast medium was power-injected through a guiding catheter located in the internal carotid artery on the affected side. The second rotation started at the delay time confirmed in earlier conventional angiography when the cerebral venous sinus was fully filled. Once the PBV map as shown in Figure 1c was acquired, an MIP image of the MCA at the occluded site (shown in Fig. 1d) was concurrently reconstructed with the use of the raw data derived from the DynaPBV Neuro. The proximal end of the occluded vessel was identified as the terminal point of the MCA where the anterograde-filling contrast medium suddenly stopped. The distal end of the occluded vessel was defined as the point nearest to the proximal end where the contrast medium retrogradely filled in the MCA through collateral vessels. The diameters at the proximal (Dp) and distal (Dd) ends were measured as shown in Figure 1d. The estimated length (L) of the occluded vessel was defined as the invariable noncontrast-enhanced distance between the proximal and distal end points of the occluded vessel on each and every arbitrary plane on the MIP image.

Mechanical thrombectomy was performed under general anesthesia in principle to prevent undesirable technical complications and to keep systolic blood pressure under $140 \mathrm{~mm} \mathrm{Hg}$ to decrease hemorrhagic transformation after reperfusion. Selection of devices for thrombectomy was left to the operator's discretion. Success in reperfusion was defined as Thrombolysis in Cerebral Infarction (TICI) grade $2 \mathrm{~b} / 3$.

The patients were divided into two groups: those with and without success in reperfusion. Baseline characteristics and each measurement value of $\mathrm{Dp}, \mathrm{Dd}$, and L were compared between the two groups. In limited cases with the use of a stent retriever, the ratio of $\mathrm{L}$ to the length of the active zone $(\mathrm{Az})$ of the stent retriever was analyzed as the L/Az ratio and was compared between the two groups.

Statistical analysis was performed using Bell Curve for Excel software (version 2.15, Social Survey Research Information Co. Ltd., Tokyo, Japan). Numerical values are expressed as median (interquartile range [IQR]), and categorical data are summarized as percentages. Dichotomous variables were analyzed using nonparametric methods: Mann-Whitney U-test and Fisher's exact test. Cut-off values were calculated using a receiver operating characteristic curve. Based on the cut-off values determined from the receiver operating characteristic curve, the odds ratio was determined by $2 \times 2$ tables and verified by Fisher's exact test. $p$ values $<0.05$ were considered to be significant.

\section{Results}

From January 2016 to December 2017, 475 patients were admitted to our hospital with a diagnosis of cerebral infarction, of whom 84 patients underwent mechanical thrombectomy for acute large-vessel occlusion, and 52 had an MCA occlusion. Finally, 29 patients examined by DynaPBV Neuro were enrolled in our study.

Table 1 presents the basic characteristics of these 29 patients, 18 of whom achieved TICI $2 \mathrm{~b} / 3$. There were no significant differences in characteristics between the two groups except for a history of hypertension. A stent retriever was used in more than two-thirds of the patients in both groups. 
Fig. 1. Representative case of a 69-year-old man with mitral valve replacement in whom anticoagulant therapy was stopped suddenly and who presented with right conjugate deviation and left hemiplegia. a DWI of MRI showing high intensity in the right corona radiate and lenticular nucleus. The ASPECTS-DWI score was 9. b Arrow indicates occlusion of the MCA on right internal carotid arteriography. c Syngo DynaPBV Neuro map was acquired by intra-aortic injection of contrast medium through a guiding catheter placed in the right internal carotid artery. d On the MIP image originating from the raw data of the syngo DynaPBV Neuro, Dp, Dd, and L were measured. e A 3-mm $\times 20$-mm Trevo XP ProVue Stentriever (Stryker, Los Angeles, CA, USA) was deployed to cover the occlusive thrombus, which was located between M1 and M2. The arrow indicates the tip of the stent retriever. $\mathbf{f}$ Successful complete reperfusion by first-pass thrombectomy. DWI, diffusion-weighted image; ASPECTS, Alberta Stroke Program Early CT Score; PBV, parenchymal blood volume; MCA, middle cerebral artery; MIP, maximum intensity projection; $\mathrm{Dp}$, diameter of proximal end; Dd, diameter of distal end; L, estimated length of distance between proximal and distal ends of the vessel occlusion.
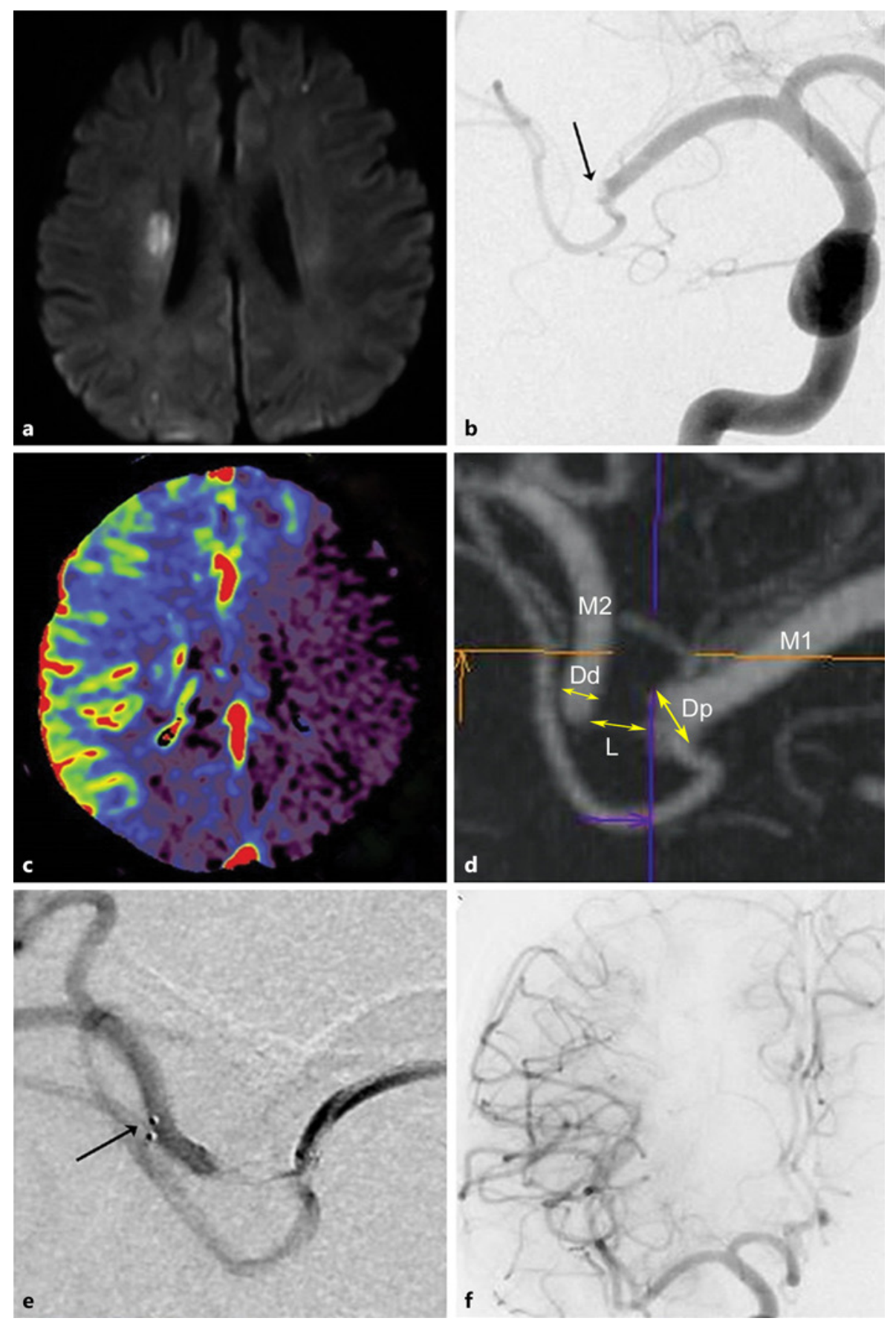

Measurements of diameters at the proximal and distal ends and the length of the occluded vessel were compared between the dichotomous groups (Table 2). In the group with TICI 2b/3, Dd (1.7 [IQR 1.5-1.9] mm) was significantly larger, and L (7.1 [IQR 4.9-9.7] $\mathrm{mm}$ ) of the occluded vessel was significantly shorter than those in the group with TICI <2b (Dd: 1.2 [IQR 1.2-1.5] mm, $p=$ 0.007 ; L: 12.3 [IQR 7.2-15.8] mm, $p=0.043$ ). Notably, the odds ratio of Dd for TICI $2 \mathrm{~b} / 3$ was 9.00 (95\% confidence interval: $1.46-55.4, p=0.02$ ).

Additionally, the L/Az ratio was analyzed in the 21 patients in whom a stent retriever was used for thrombec- 
Table 1. Baseline patient characteristics

\begin{tabular}{lllll}
\hline Characteristic & $\begin{array}{l}\text { Total } \\
(n=29)\end{array}$ & $\begin{array}{l}\text { TICl: 0, 1, 2a } \\
(n=11)\end{array}$ & $\begin{array}{l}\text { TICl: } 2 \mathrm{~b}, 3 \\
(n=18)\end{array}$ & $p$ value \\
\hline Age, median (IQR), years & $73(69-79)$ & $73(70-77)$ & $74(68-78)$ & 0.857 \\
Female sex, $n$ (\%) & $10(34)$ & $3(27)$ & $7(38)$ & 0.165 \\
Atrial fibrillation, $n$ (\%) & $17(58)$ & $7(63)$ & $10(55)$ & 0.534 \\
Diabetes mellitus, $n$ (\%) & $1(3)$ & 0 & $1(5)$ & 1.000 \\
Dyslipidemia, $n$ (\%) & $9(31)$ & $5(45)$ & $4(22)$ & 0.237 \\
Hypertension, $n$ (\%) & $15(51)$ & $10(90)$ & $5(27)$ & 0.003 \\
Pretreatment of antithrombotic medication, $n(\%)$ & $16(55)$ & $6(54)$ & $10(55)$ & 1.000 \\
NIHSS score, median (IQR) & $16(11-20)$ & $15(11-18)$ & $19(13-20)$ & 0.302 \\
DWI-ASPECTS, median (IQR) & $7(4-8)$ & $7(5-8)$ & $5(4-8)$ & 0.716 \\
M1 occlusion, $n$ (\%) & $15(51)$ & $4(36)$ & $11(61)$ & 0.362 \\
Intravenous injection of alteplase, $n$ (\%) & $5(17)$ & $2(18)$ & $3(16)$ & 1.000 \\
Onset-to-groin puncture time, median (IQR), min & $224(201-246)$ & $237(222-246)$ & $215(194-240)$ & 0.101 \\
Onset-to-reperfusion time, median (IQR), min & $\mathrm{NA}$ & $\mathrm{NA}$ & $294(249-303)$ & $\mathrm{NA}$ \\
Stent retriever, $n$ (\%) & $21(72)$ & $9(81)$ & $12(66)$ & 0.67 \\
Length of active zone, median (IQR), mm & $20(20-24)$ & $20(18-21)$ & $20(20-22)$ & 0.271 \\
\hline
\end{tabular}

$\mathrm{TICl}$, Thrombolysis in Cerebral Infarction score; IQR, interquartile range; NIHSS, National Institutes of Health Stroke Scale; DWI, diffusion-weighted image; ASPECTS, Alberta Stroke Program Early CT Score; NA, not applicable. The $p$ value was tested using Fisher's exact test for categorical data and the Mann-Whitney U-test for other variables.

Table 2. Median measurements of the occluded vessel and ROC curve cut-off points in patients with or without reperfusion and in those with stent retriever use

\begin{tabular}{|c|c|c|c|c|c|c|c|}
\hline Variable & $\begin{array}{l}\text { TICl: } 0,1,2 \mathrm{a} \\
\text { (IQR), mm }\end{array}$ & $\begin{array}{l}\text { TICl: } 2 b, 3 \\
\text { (IQR), mm }\end{array}$ & $p$ value & $\begin{array}{l}\text { Optimal } \\
\text { cut-off value }\end{array}$ & $\begin{array}{l}\text { AUC } \\
\text { for ROC }\end{array}$ & Sensitivity & Specificity \\
\hline Patients with/without reperfusion & $n=11$ & $n=18$ & & & & & \\
\hline Dd & $1.2(1.2-1.5)$ & $1.7(1.5-1.9)$ & 0.007 & 1.6 & 0.80 & 0.66 & 0.81 \\
\hline $\mathrm{L}$ & $12.3(7.2-15.8)$ & $7.1(4.9-9.7)$ & 0.043 & 8.5 & 0.72 & 0.72 & 0.63 \\
\hline Patients with/without reperfusion + stent retriever use & $n=9$ & $n=12$ & & & & & \\
\hline
\end{tabular}

$\mathrm{TICl}$, Thrombolysis in Cerebral Infarction score; IQR, interquartile range; AUC, area under the curve; ROC, receiver operating characteristic; NA, not applicable; Dp, proximal diameter; $\mathrm{Dd}$, distal diameter; $\mathrm{L}$, estimated length of distance between proximal and distal ends of the vessel occlusion

tomy (Table 2). Of them, 12 patients achieved TICI $2 \mathrm{~b} / 3$ and showed a significantly smaller $\mathrm{L} / \mathrm{Az}$ ratio $(0.31[\mathrm{IQR}$ $0.21-0.34])$ than the other 9 patients with TICI $<2 \mathrm{~b}(0.61$ [IQR 0.40-0.72]; $p=0.002)$, although there was no significant difference between the dichotomized groups in the length of the active zone of the stent retrievers used (Table 1). The cut-off value of the L/Az ratio for TICI $2 \mathrm{~b} / 3$ was 0.32 . Images from a representative case are shown in Figure 1.

\section{Discussion/Conclusion}

We found that the diameter and length of an occluded vessel on MIP images acquired by FD-CT are substantially relevant to the achievement of TICI $2 \mathrm{~b} / 3$ following mechanical thrombectomy in patients with acute MCA occlusion.

This study found that a cut-off value of estimated length of the occluded vessel of $8.5 \mathrm{~mm}$ might indicate achievement of a higher rate of TICI $2 \mathrm{~b} / 3$. In our proto- 
col, the second rotation of FD-CT is run in the late venous phase when the venous sinus is fully filled with contrast medium as confirmed by earlier conventional angiography. We consider that the contrast medium can retrogradely reach closely enough to the distal end of the occluded vessel on the MIP image. From our literature search, we could find no reports discussing relevance between the length of the occluded vessel and achievement of favorable reperfusion following thrombectomy, although there are some reports on estimating the length of an occlusive thrombus in acute large-vessel occlusion so as to chiefly distinguish cardiogenic embolism from other etiologies. Zhou et al. [5] reported that cardiac embolism may occur when the length of the delayed contrast filling sign of an intra-arterial clot on CT angiography is longer than $1.5 \mathrm{~mm}$, which seemed to be a partial length of the vessel occlusion. Zhang et al. [6] reported that overestimation of the length (cut-off value: $10 \mathrm{~mm}$ ) of the occlusive thrombus as the susceptibility vessel sign on MRI suggests cardiogenic embolus. Moreover, we elucidated via sub-analysis that the length of the active zone of a stent retriever that is more than 3 times longer than that of the occluded vessel might be another predictive factor for successful reperfusion. We consider that a stent retriever might be easy to deploy and is sure to be effective as its active zone is long enough to cover the full length of the occlusive thrombus. Haussen et al. [10] reported that the use of longer stent retrievers (Solitaire $4 \times 40 \mathrm{~mm}$, Trevo $4 \times 30 \mathrm{~mm}$ ) is an independent predictor of first-pass modified TICI $2 \mathrm{~b} / 3$ reperfusion, which supports our findings. They considered that longer stent retrievers may allow precise placement by increasing the margin of error to fully cover the occlusive thrombus.

We also showed that a larger diameter at the distal end of the vessel occlusion might be another indicator of favorable reperfusion following mechanical thrombectomy. A large diameter at the distal end of the occluded vessel may allow a stent retriever to easily expand sufficiently during deployment even at the distal tip and grasp an occlusive thrombus firmly and, finally, to make it more difficult to release the thrombus during stent withdrawal.

In the group with unsuccessful reperfusion, $90 \%$ of the patients had a history of hypertension (Table 1) and longer length of vessel occlusion (Table 2). In such cases, partly because chronic atherosclerotic stenosis might have previously existed at the occlusion site, other procedures besides thrombectomy should be considered, such as percutaneous transluminal balloon angioplasty [11] or urgent extracranial-intracranial bypass surgery [12].
This study has limitations inherent to a single-center retrospective observational analysis with a small sample size. In acute occlusion of the internal carotid artery, it is difficult to apply the same approach to estimate the distal end of an occluded vessel because collateral flow is complicated and supplied by several salvage pathways such as the anterior/posterior communicating artery or ophthalmic artery.

This study clearly showed that both larger diameter of the distal end and shorter length of an acute MCA occlusion might have significant relevance in attaining TICI $2 \mathrm{~b} / 3$ reperfusion following mechanical thrombectomy. Moreover, a sub-analysis clarified that a stent retriever with an active zone length long enough to cover the vessel occlusion might be another factor predictive of favorable reperfusion.

\section{Statement of Ethics}

This study protocol was reviewed and approved by the Ethics Review Board of Nagareyama Central Hospital (approval number $\mathrm{NNN}$ ). Subjects (or their parents or guardians) have given their written informed consent.

\section{Conflict of Interest Statement}

The authors have no conflicts of interest to declare.

\section{Funding Sources}

This study did not receive any funding.

\section{Author Contributions}

T.Y. designed, organized, and conducted the study and wrote the manuscript. T.U., T.H., H.O., N.A., and Y.U. obtained data. T.Y. and R.K. critically reviewed the manuscript.

\section{Data Availability Statement}

All data generated or analyzed during this study are included in this article. Further enquiries can be directed to the corresponding author. 


\section{References}

1 Berkhemer OA, Fransen PS, Beumer D, van den Berg LA, Lingsma HF, Yoo AJ, et al. A randomized trial of intraarterial treatment for acute ischemic stroke. N Engl J Med. 2015 Jan 1;372(1):11-20.

2 Protto S, Sillanpää N, Pienimäki JP, Matkaselkä I, Seppänen J, Numminen H. Stent retriever thrombectomy in different thrombus locations of anterior cerebral circulation. Cardiovasc Intervent Radiol. 2016 Jul;39(7):98893.

3 Schwaiger BJ, Gersing AS, Zimmer C, Prothmann S. The curved MCA: influence of vessel anatomy on recanalization results of mechanical thrombectomy after acute ischemic stroke. AJNR Am J Neuroradiol. 2015 May; 36(5):971-6.

4 Bang OY, Saver JL, Kim SJ, Kim GM, Chung CS, Ovbiagele B, et al. Collateral flow predicts response to endovascular therapy for acute ischemic stroke. Stroke. 2011 Mar;42(3):6939.
5 Zhou Y, Xu C, Zhang R, Shi F, Liu C, Yan S, et al. Longer length of delayed-contrast filling of clot on 4-dimensional computed tomographic angiography predicts cardiogenic embolism. Stroke. 2019 Sep;50(9):2568-70.

6 Zhang R, Zhou Y, Liu C, Zhang M, Yan S, Liebeskind DS, et al. Overestimation of susceptibility vessel sign: a predictive marker of stroke cause. Stroke. 2017 Jul;48(7):1993-6.

7 Struffert T, Deuerling-Zheng Y, Kloska S, Engelhorn T, Strother CM, Kalender WA, et al. Flat detector CT in the evaluation of brain parenchyma, intracranial vasculature, and cerebral blood volume: a pilot study in patients with acute symptoms of cerebral ischemia. AJNR Am J Neuroradiol. 2010 Sep;31(8): 1462-9.

8 Struffert T, Deuerling-Zheng Y, Kloska S, Engelhorn T, Lang S, Mennecke A, et al. Dynamic angiography and perfusion imaging using flat detector CT in the angiography suite: a pilot study in patients with acute middle cerebral artery occlusions. AJNR Am J Neuroradiol. 2015 Oct;36(10):1964-70.
9 Minematsu K, Toyoda K, Hirano T, Kimura K, Kondo R, Mori E, et al. Guidelines for the intravenous application of recombinant tissue-type plasminogen activator (alteplase), the second edition, October 2012: a guideline from the Japan Stroke Society. J Stroke Cerebrovasc Dis. 2013 Jul;22(5):571-600.

10 Haussen DC, Al-Bayati AR, Grossberg JA, Bouslama M, Barreira C, Bianchi N, et al. Longer stent retrievers enhance thrombectomy performance in acute stroke. J Neurointerv Surg. 2019 Jan;11(1):6-8.

11 Baek JH, Kim BM, Heo JH, Kim DJ, Nam HS, Kim YD. Outcomes of endovascular treatment for acute intracranial atherosclerosisrelated large vessel occlusion. Stroke. 2018 Nov;49(11):2699-705.

12 Nussbaum ES, Janjua TM, Defillo A, Lowary JL, Nussbaum LA. Emergency extracranialintracranial bypass surgery for acute ischemic stroke. J Neurosurg. 2010 Mar;112(3):666-73. 